\title{
¿Hacia una mayor influencia? Análisis de la participación negociadora de países en desarrollo durante la Ronda Doha ${ }^{1}$
}

Towards a greater influence? Analysis of developing countries' participation during the Doha Round

Julieta Zelicovich ${ }^{2}$

\section{RESUMEN}

Tradicionalmente las negociaciones comerciales multilaterales habían sido protagonizadas por los países desarrollados. En la Ronda Doha se identifica un conjunto de países en desarrollo, que de manera creciente han tenido una participación cada vez más activa, llegando a alcanzar considerables niveles de influencia. El objetivo de este artículo es indagar en las particularidades de la participación de estos Estados, a partir de un análisis comparado.

Palabras claves: Negociaciones Comerciales; Países en Desarrollo; Ronda Doha

\begin{abstract}
Traditionally, multilateral trade negotiations had been protagonized by developed countries. During the Doha Round, we identify a group of developing countries that has incremented the activism of its participation, reaching considerable levels of influence. The aim of this paper is to study the particularities of the participation of those states, from a comparative analysis point of view.
\end{abstract}

Keywords: Trade Negotiations; Developing Countries; Doha Round

Desde la creación del Acuerdo General de Comercio y Aranceles (conocido como GATT, por sus siglas en inglés) las negociaciones de comercio multilaterales han estado dominadas por los países desarrollados. Durante décadas habían sido éstos quienes establecieron los contenidos de la agenda de negociación, las modalidades de acuerdo, y los alcances de su aplicación; mientras que los países en desarrollo mantenían una participación periférica, al menos hasta la Ronda Uruguay (Tussie, 1993; VanGrasstek, 2013).

\footnotetext{
${ }^{1}$ Artigo recebido em 26 de março de 2014 e aprovado em 31 de março de 2014.

${ }^{2}$ Doctora en Relaciones Internacionales. Universidad Nacional de Rosario. Rosario, Argentina.

Conjuntura Global, Vol.3, n.1, jan./mar., 2014, p. 03-12.
} 
La participación no significa solamente ser parte formal del proceso negociador, sino que implica una actuación real: el desarrollo de un conjunto de herramientas y acciones (como la presencia en instancias claves, la presentación de documentos, la conformación de grupos de presión, etc.) que conduzcan a buscar influenciar en la adopción de decisiones dentro de la negociación, conforme a los propios objetivos (Peña, 1968). Conforme señala Peña el desafío es "[tener y utilizar] los medios adecuados para logar el ingreso en los mecanismos reales de participación e influenciar así en la adopción de aquellas decisiones que afectan su vida externa” (Peña, 1968: 24-27). Se requiere tanto de las capacidades y habilidades de los Estados, como del marco del proceso de toma de decisión donde se insertan (Tussie y Lengyel, 2002).

En la Ronda de Doha los países emergentes han tenido tales capacidades, alcanzando un protagonismo clave ${ }^{3}$. No obstante, a la par de estos actores existió un segundo conjunto de Estados, países en desarrollo, que de manera creciente han tenido una participación cada vez más activa en las negociaciones, llegando a alcanzar considerables niveles de influencia. El objetivo de este artículo es indagar en las particularidades de la participación de estos Estados.

Para ello se seleccionaron 11 países que han mantenido constante su participación en los flujos del comercio mundial. A partir de la información disponible en la base de datos de la OMC, se ha procedido a elaborar una serie de indicadores comparados sobre la participación de los mismos en la Ronda Doha. Las ideas que se busca demostrar son que los países en desarrollo están generando una participación creciente en las negociaciones comerciales multilaterales; y que dentro del conjunto de países en desarrollo Argentina ha sido un negociador destacado por su activismo.

\section{Las negociaciones de comercio: una arena asimétrica}

La historia reciente del comercio mundial expresa una alta concentración de los flujos del intercambio comercial en pocas economías. Cinco economías explican el 50\% del comercio mundial, en tanto que son necesarios sólo veinticinco países para explicar el 88\% de los intercambios de bienes a nivel global (OMC, 2012). Esta concentración no solo ha impactado en el grado de desarrollo de los Estados, sino que además ha ido

${ }^{3}$ Véase Messerlin, 2004

Conjuntura Global, Vol.3, n.1, jan./mar., 2014, p. 03-12. 
afectando la evolución de las negociaciones. Si bien tanto en el GATT como en la OMC ha primado el criterio de un estado igual a un voto, las relaciones de poder han atravesado las prácticas de negociación desde sus orígenes, enfatizando las asimetrías en la negociación multilateral.

En la historia del GATT uno de los mecanismos de toma de decisión por el cual se consolidó la participación asimétrica en las negociaciones fue la práctica del principio del "proveedor dominante" (Tussie, 1993:36). Acorde al mismo las negociaciones se hacían de manera bilateral y por listas de productos específicos, entre los exportadores e importadores de mayor volumen y luego eran extendidas al resto a través de la cláusula de Nación Más favorecida. Este principio permitía expandir los alcances de las reducciones arancelarias, pero tendiendo a mantener las estructuras de participación relativa de cada uno de los Estados dentro de los flujos del comercio mundial, de manera que los principales proveedores de cada producto conservaban su status de poder de mercado.

A la par que se desarrollaban estas costumbres, otras prácticas restrictivas de la participación de los países en desarrollo han sido las instancias informales de negociación. Si bien la OMC prevé mecanismos de reunión donde todos los Estados están representados, lo cierto es que desde la dirección general del organismo se ha tendido al desarrollo de reuniones informales y consultas parciales con determinados actores países desarrollados y países emergentes-, dejando de lado a la mayoría de los miembros, no desarrollados. Tanto en el acceso a estas reuniones, como en la circulación de tales propuestas, se denuncia la existencia de gran discrecionalidad en el manejo de la información. Ello pues empoderaba a los países desarrollados, y limitaba las posibilidades de las delegaciones de los países en desarrollo, que se ven excluidas.

Otros mecanismos de asimetría señalados en la literatura han sido los del lenguaje (Kwa, 2003), así como los de la amplitud de la agenda negociadora (Steinberg, 2007). En ambos casos los mecanismos de aprendizaje y la adquisición de experiencia negociadora resultaban fundamentales para sobrepasar los diversos obstáculos presentados. 
En efecto, fue sólo cuando los países en desarrollo, amparados en el trato especial y diferenciado 4 fueron adquiriendo tal experiencia, que su participación real en las negociaciones comenzó a incrementarse. La Conferencia de la UNCTAD de los 60, y la Ronda de Uruguay, fueron procesos en los cuales las voces de estos países comenzaron a hacer eco en las instituciones del régimen internacional de comercio. Tener mayor participación significó salir del rol pasivo de ser meramente un rule-taker, sin embargo el mayor involucramiento no implicó en forma directa mayor efectividad o resultados en el acceso al mercado global (Tussie y Lengyel, 2002). Recién en la Ronda Doha los países emergentes y países en desarrollo han ganado verdadero protagonismo, con experiencias como la de la coalición del G20, el COTTON-4.

\section{La participación de los países en desarrollo en la Ronda Doha}

Después de los intentos de lanzar una nueva ronda de negociaciones en Seattle, y bajo la necesidad de consolidar el multilateralismo frente a los atentados del 11-S, los países desarrollados acordaron en 2001, un paquete de temas que favorecerían a los países en desarrollo dentro de las negociaciones de OMC. Nació así la Ronda Doha, denominada Ronda del Desarrollo. Estos cambios en la composición de la agenda provocaron que ciertos países, que no habían sido protagonistas centrales de las negociaciones anteriormente, generasen considerables niveles de participación, buscando influenciar en la definición de los nuevos marcos normativos que regularían el comercio a nivel multilateral, especialmente en agricultura y bienes industriales.

Estos países presentaron rasgos distintos, tanto de los países desarrollados, como de las potencias emergentes, que estaban comenzando a hacer sentir su peso en los diferentes ámbitos de la gobernanza global. Se trataba de Estados medios, medianos o con poderes moderados; es decir, aquellos que "al igual que los estados pequeños, (...) carecen del peso militar o político que generalmente se necesita para ser influyente en forma unilateral. Pero, a diferencia de los estados pequeños, (...) suelen tener los recursos necesario para participar activamente en organizaciones internacionales, como un cuerpo diplomático bien entrenado, una red extensa de embajadas y misiones diplomáticas y personal preparado en el país que puede colaborar con el que trabaja en

\footnotetext{
${ }^{4}$ Véase Peixoto, 2010
}

Conjuntura Global, Vol.3, n.1, jan./mar., 2014, p. 03-12. 
el exterior." (Miller, 2000: 41). Entre estos países pueden considerarse a Argentina, Nigeria, Chile, entre otros, que han seguido este camino de incremento en la participación en la búsqueda de mayor influencia.

Nuestro estudio partió de la identificación de tales países, los cuales podían ser considerados países medios en materia de las relaciones comerciales internacionales. Para ello se seleccionaron los casos que a lo largo de la década se mantuvieron entre el puesto 30 y 40 del ranking global de exportadores de bienes, y que han contribuido al 0,5 del comercio global. El resultado fue la identificación de un conjunto heterogéneo de 11 países. Como puede verse en la Tabla 1, se trata de economías entre medianas y pequeñas, con diferente distribución del ingreso. Se basan en manufacturas de origen industrial, o en combustibles y minerales, siendo Argentina el único de este conjunto de países que centra su participación en el comercio mundial en los productos agrícolas. En ninguno de los casos se trata de productores hegemónicos, ni que tengan participación en cuotas de mercado mayores al 3\%. Su pertenencia regional es variada, abarcando tanto Sudamérica, como Europa, Asia y Medio Oriente.

TABLA 1: Países medios en las negociaciones OMC

\begin{tabular}{|l|r|r|l|l|l|}
\hline & $\begin{array}{l}\text { Participación } \\
\text { del comercio } \\
\text { global - 2001 }\end{array}$ & $\begin{array}{l}\text { Participación } \\
\text { del comercio } \\
\text { global - 2010 }\end{array}$ & $\begin{array}{c}\text { Principal producto } \\
\text { exportado }\end{array}$ & $\begin{array}{l}\text { Partipación del } \\
\text { comercio global } \\
\text { del principal } \\
\text { producto } \\
\text { exportado (2012) }\end{array}$ & $\begin{array}{c}\text { PBI } \\
\text { (millones } \\
\text { de US\$, } \\
2010)\end{array}$ \\
\hline ARGENTINA & 0,4 & 0,4 & Productos agrícolas & $3 \%$ & 368736 \\
\hline NIGERIA & 0,3 & 0,5 & Combustibles y Minerales & $3 \%$ & 193669 \\
\hline SUDÁFRICA & 0,5 & 0,5 & Manufacturas & $0 \%$ & 363704 \\
\hline VIETNAM & 0,2 & 0,5 & Manufacturas & 106427 \\
\hline CHILE & 0,3 & 0,5 & Combustibles y Minerales & $1 \%$ & 212741 \\
\hline FINLANDIA & 0,7 & 0,5 & Manufacturas & $0 \%$ & 238746 \\
\hline KUWAIT & 0,3 & 0,4 & Combustibles y Minerales & $3 \%$ & 109463 \\
\hline VENEZUELA & 0,4 & 0,4 & Combustibles y Minerales & $2 \%$ & 391847 \\
\hline REP. ESLOVACA & 0,2 & 0,4 & Manufacturas & $1 \%$ & 87268 \\
\hline QATAR & - & 0,4 & Combustibles y Minerales & $3 \%$ & 98313 \\
\hline ISRAEL & 0,4 & Manufacturas & $1 \%$ & 217333 \\
\hline
\end{tabular}

Fuente: elaboración propia en base a datos OMC 
Dentro de este conjunto han sido han sido relevados diversos desempeños en las negociaciones. Países que han sido activos en todos los temas, países con intereses segmentados, países que han priorizado la presentación de documentos, mientras que otros han centrado su accionar en las coaliciones negociadoras (Tabla 2). Lo que la comparación sugiere es que, a diferencia de lo que sucede para los países centrales del comercio global, entre los países en desarrollo una participación semejante en los flujos del comercio mundial no equivale a una participación equivalente en las negociaciones internacionales de comercio. Así, cuando la participación en los intercambios de bienes no genera posiciones de oligopolio u oligopsonio en los mercados, tal variable no parecería resultar un valor o indicador suficiente de la conducta de los Estados en los procesos de negociación. Asimismo, las diferencias señaladas permiten inferir una segunda afirmación, y es que los países en desarrollo, países medios, aún si resultan crecientemente más activos difícilmente puedan considerarse como un conjunto o grupo negociador en cuanto a sus prácticas negociadoras.

TABLA 2. Indicadores de participación en las negociaciones multilaterales de comercio 2001-2011

\begin{tabular}{|c|c|c|c|c|c|c|c|c|}
\hline & \multirow[b]{2}{*}{$\begin{array}{c}\text { Participación } \\
\text { en } \\
\text { coaliciones } \\
\text { negociadoras }\end{array}$} & \multicolumn{4}{|c|}{ Presentación de Documentos } & \multirow[b]{2}{*}{$\begin{array}{l}\text { Asistencia a } \\
\text { reuniones } \\
\text { mini- } \\
\text { ministeriales }\end{array}$} & \multirow[b]{2}{*}{$\begin{array}{c}\text { Presidencias } \\
\text { y/o } \\
\text { direcciones } \\
\text { o co- } \\
\text { direcciones }\end{array}$} & \multirow[b]{2}{*}{$\begin{array}{c}\text { Participación } \\
\text { en el ESD }\end{array}$} \\
\hline & & 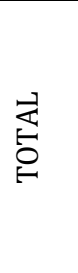 & 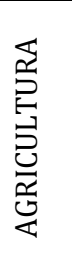 & $\sum^{\ll}$ & 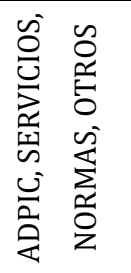 & & & \\
\hline ARGENTINA & 6 & 77 & 19 & 34 & 24 & 6 & NO & 73 \\
\hline NIGERIA & 7 & 7 & 1 & 4 & 2 & 4 & SI & 1 \\
\hline SUDÁFRICA & 8 & 29 & 5 & 15 & 9 & 9 & SI & 6 \\
\hline VIETNAM & 3 & 8 & 1 & 1 & 6 & 0 & NO & 14 \\
\hline CHILE & 5 & 101 & 2 & 9 & 90 & 4 & SI & 56 \\
\hline FINLANDIA & 3 & 1 & 0 & 0 & 1 & 0 & NO & 0 \\
\hline KUWAIT & 1 & 0 & 0 & 0 & 0 & 0 & NO & 1 \\
\hline VENEZUELA & 3 & 33 & 2 & 15 & 16 & 0 & NO & 19 \\
\hline REP. ESLOVACA & 3 & 3 & 0 & 0 & 3 & 0 & NO & 3 \\
\hline QATAR & 1 & 4 & 0 & 3 & 1 & 0 & NO & 0 \\
\hline ISRAEL & 3 & 41 & 3 & 1 & 37 & 0 & NO & 4 \\
\hline
\end{tabular}

Fuente: Elaboración propia en base a datos OMC 
La participación en coaliciones negociadoras ha sido una de las tácticas de negociación más utilizada por este grupo. Es a partir de la agregación de esfuerzos y la adopción de estrategias integrativas que estas coaliciones implican, que los países en desarrollo pueden incrementar su participación, con mayor peso de mercado, mayores redes de inteligencia comercial, y mayores oportunidades para llegar acuerdos satisfactorios con terceros (Drahos, 2003). Los países africanos, Nigeria y Sudáfrica son los que más intensamente apelaron a este mecanismo. En el detalle de las coaliciones a las que estos países pertenecen se observa la combinación de coaliciones de base regional (ej. Países africanos), con coaliciones temáticas (Patrocinadores del documento w52) y coaliciones de "nuevo tipo" 5 " (como el G20). En el resto de los casos se observa en cambio la primacía de coaliciones temáticas, específicas ${ }^{6}$.

En cuanto a la presentación de documentos todos los países seleccionados, con la excepción de Kuwait, han sido activos promotores de esta táctica. Chile resulta el actor más destacado, seguido por Argentina e Israel. Mientras que en el primero de los casos hay un equilibrio entre los tres grandes temas de negociación, Israel y Chile tienen una mayor preocupación por la presentación de documentos en cuestiones normativas no arancelarias, no vinculadas a las negociaciones de AMNA ni a las de agricultura.

La participación en las reuniones miniministeriales es un reconocimiento de los pares hacia la importancia del país en el desarrollo de la negociación. En este caso, durante la Ronda Doha, solo 4 de los países considerados participaron de las diversas reuniones informales y miniministeriales que se sucedieron. Los mismos fueron Argentina, Nigeria, Sudáfrica y Chile. En el primero de los casos la particiapcion en estas instancias puede deberse al carácter central que la Argentina tiene en el comercio agrícola y a su activa presentación de documentos. Chile también ha de explicar parte de tal participación en el activismo observado a la hora de presentar proyectos. Nigeria y Sudáfrica por su parte tienen un peso importante como representantes de las diversas coaliciones que conforman.

El último de los indicadores que se consideró ha sido la participación dentro del entendimiento de solución de diferencias y la presencia de presidentes, co-presidentes y

\footnotetext{
${ }^{5}$ Vease Narlikar 2005

6 Un listado actualizado de las coaliciones negociadoras durante la Ronda Doha puede consultarse en http://wto.org/english/tratop_e/dda_e/negotiating_groups_e.htm
}

Conjuntura Global, Vol.3, n.1, jan./mar., 2014, p. 03-12. 
chairs dentro de la OMC, oriundos de los países en cuestión. Si bien estos indicadores no se relacionan en forma directa con las negociaciones de Doha, son tomados aquí como un indicador general de participación dentro del régimen multilateral de comercio. Los datos analizados permiten ver que muy pocos países del conjunto han tenido un papel de reguladores en las negociaciones, manteniéndose en este aspecto la tendencia señalada en la historia de la OMC, donde los espacios de protagonismo eran asignados a los países desarrollados. En cuanto al ESD existe un paulatino involucramiento de los países en desarrollo en este procedimiento. Argentina, Chile y Venezuela tienen un mayor grado de participación que el resto. Conforme estos datos, se coincide con Shaffer, et al. (2008) en que hay una vinculación entre la participación institucional, especialmente dentro del proceso de solución de diferencias, y la creación de capacidades para las negociaciones en el plano multilateral.

Tal como afirmamos, existe una participación creciente de los países en desarrollo "medios" o "medianos" en las negociaciones multilaterales de comercio, pero ésta no resulta homogénea. Puede identificarse un núcleo más activo dentro de los 11 países seleccionados, y en particular, un caso, el argentino, resulta más llamativo.

\section{Argentina: ¿un negociador destacado?}

A partir de los indicadores considerados en este artículo, resulta posible afirmar que Argentina ha desarrollado una conducta destacada en las negociaciones de Ronda Doha, por sobre otros países con participaciones similares en el comercio mundial. Sudáfrica y Chile se aproximan bastante, uno por su participación en las reuniones mini ministeriales, y el otro por una activa presentación de documentos en las negociaciones de normas para las medidas de antidumping.

No obstante, el equilibrio entre los diferentes aspectos de la conducta negociadora de la Argentina es lo que hace que pueda ser considerada por encima de estos otros, convirtiéndose en un "overachiever" de las negociaciones comerciales. Este concepto tomado de un artículo de Walt (2009), remite a aquellos que "perform better or achieve more sucess than expected". Sugerimos entonces que la Argentina en determinados momentos de la negociación -cuando confluyen los distintos indicadores considerados- presenta comportamientos mayores a los esperados.

Conjuntura Global, Vol.3, n.1, jan./mar., 2014, p. 03-12. 


\section{Bibliografía}

DRAHOS, Peter. When the weak bargain with the strong: Negotiations in the World Trade Organization" International Negotiation, Nueva York, n 8. 2003.

KWA, A. Power Politics in the WTO. Bangkok, Tailandia: Ed. Focus on the Global South. 2003.

MESSERLIN, Patrick. G2 and G20, Please Tango!. 2004. (http://www.iai.it/pdf/DocIAI/iai0412e.pdf consultado en octubre de 2008)

MILLER, Carina., Influencia sin poder. El desafío argentino ande los foros internacionales. Buenos Aires: Grupo Editor Latinoamericano. 2000

NARLIKAR, Amrita. Bargaining over the Doha Development Agenda: Coalitions in the WTO. LATN Working Paper, Buenos Aires, Latin American Trade Network. 2005.

OMC. Estadísticas del comercio internacional $2012 . \quad 2012$ (http://www.wto.org/english/res e/statis e/its2012 e/its12 toc e.htm junio 2013)

PEIXOTO, Juliana. Flexibilidades para países en desarrollo en la ronda Doha: el Trato Especial y Diferenciado a la carta. (http://catedraomc.flacso.org.ar/wpcontent/uploads/2012/02/Anexo-2_Flexibilidades-para-Pa\%C3\%ADses-en-

Desarrollo.pdf marzo de 2011)

PEÑA, Felix (1968) "La participación en el sistema internacional” en PEÑA, Félix (2003) Momentos y Perspectivas. La argentina en el mundo y en América Latina. Buenos Aires: Editorial de la Universidad Nacional de Tres de Febrero

SHAFFER, et al. Winning at the WTO: The development of a trade policy community within Brazil. Area RRII Flacso/Argentina, Documento de trabajo $\mathrm{n}^{\circ} 14.2008$ (http://rrii.flacso.org.ar/wp-content/uploads/2008/06/doc14.pdf Junio 2010)

STEINBERG, Federico. Cooperación y conflicto. Madrid: Ed. AKAL. 2007

TUSSIE, Diana y LENGYEL, Miguel. Developing Countries: Turning Participation into Influence. En HOEKMAN, Bernard, MATTO0, Aaditya, y ENGLISH Philip, Development, Trade, and the WTO. Washington, D.C.: World Bank. 2002. 
TUSSIE, Diana. Los países menos desarrollados y el sistema de comercio mundial, México, D.F.: Fondo de Cultura Económica. 1993 (1986).

VANGRASSTEK, Craig. The History and Future of the World Trade Organization. Ginebra: WTO Publications. 2013

WALT, Stephen. "Over-achievers and under-achivers" en Foreign Policy, voice, 21/04/2009. (http://www.foreignpolicy.com/posts/2009/04/21/ over_achievers_ and_under_achievers) 